\title{
Angular Magnetoresistance of Nanowires with Alternating Cobalt and Nickel Segments
}

\author{
H. Mohammed ${ }^{1}$, H. Corte-León ${ }^{2}$, Y. P. Ivanov ${ }^{1,3,4}$, J. A. Moreno $^{1}$, O. Kazakova ${ }^{2}$, and J. Kosel ${ }^{1}$ \\ ${ }^{1}$ King Abdullah University of Science and Technology, Electrical Engineering Department, Thuwal, Saudi Arabia \\ ${ }^{2}$ National Physical Laboratory, London, United Kingdom \\ ${ }^{3}$ Austrian Academy of Sciences, Erich Schmid Institute of Materials Science, Leoben, Austria \\ ${ }^{4}$ School of Natural Sciences, Far Eastern Federal University, Vladivostok, Russia
}

\begin{abstract}
Magnetization reversal in segmented $\mathrm{Co} / \mathrm{Ni}$ nanowires with varying number of segments was studied using angular Magnetoresistance (MR) measurements on isolated nanowires. The MR measurements offer an insight into the pinning of domain walls within the nanowires. Angular MR measurements were performed on nanowires with two and multiple segments by varying the angle between the applied magnetic field and nanowire $\left(-90^{\circ} \leq \theta \leq 90^{\circ}\right)$. The angular MR measurements reveal that at lower values of $\theta$ the switching fields are nearly identical for the multisegmented and two-segmented nanowires, whereas at higher values of $\theta$, a decrease in the switching field is observed in the case of two segmented nanowires. The two segmented nanowires generally exhibit a single domain wall pinning event, whereas an increased number of pinning events are characteristic of the multisegmented nanowires at higher values of $\theta$. In-situ magnetic force microscopy substantiates reversal by domain wall nucleation and propagation in multisegmented nanowires.
\end{abstract}

\section{Index Terms-Cylindrical Nanowires, Angular Magnetoresistance, Domain Wall Pinning, Magnetic Force Microscopy}

\section{INTRODUCTION}

$\mathrm{T}^{\mathrm{s}}$ he prospect of utilizing the vertical dimension to enhance data storage capacity has spurred considerable interest in developing 3D memory devices [1]-[3]. In particular, cylindrical magnetic nanowires are emerging as attractive $3 \mathrm{D}$ architectures that provide a low cost and simple fabrication technique where nanowires are electrochemically grown parallel to each other in a vertical fashion [4]. In addition to 3D scaling, the non-planarity of nanowires has been theoretically predicted to be advantageous to the motion of domain walls (DWs) [5]. Even though cylindrical nanowires appear as attractive candidates, their realization has been hindered due to the difficulties in achieving reliable pinning sites as well as the challenges involved in performing single nanowire-based studies. The realization of these 3D devices would depend on the demonstration of moving DWs from one pinning site to another. Approaches such as diameter modulation and multisegmented nanowires, providing pinning sites for DWs, have been demonstrated [6]-[8]. Here, we investigate the segmented nanowires consisting of alternating segments of cobalt and nickel, wherein the interfaces act as pinning sites for domain walls. For the realization of a multisegmented nanowire-based device, an understanding of the magnetization reversal mechanism as well as angular switching field dependence is important. In this paper, we perform Magnetoresistance (MR) measurements to study the magnetization reversal and angular switching field dependence in nanowires with different numbers of $\mathrm{Co} / \mathrm{Ni}$ segments. In addition to this, Magnetic Force Microscopy (MFM) has been employed to investigate the mechanism of domain wall nucleation and propagation in segmented $\mathrm{Co} / \mathrm{Ni}$ nanowires.

\section{METHODS}

\section{A. Fabrication of Cylindrical Nanowires}

Cylindrical nanowires were grown by electrodeposition into Anodic Aluminum Oxide (AAO) templates with pore diameter $(\phi)$ of approximately $80 \mathrm{~nm}$ and inter pore distance of $105 \mathrm{~nm}$. The AAO templates were fabricated using two subsequent anodization processes in oxalic acid solution (see [7] for more details). Segmented $\mathrm{Co} / \mathrm{Ni}$ nanowires were fabricated by electrodeposition into the AAO templates using two separate solutions. Electrodeposition of $\mathrm{Ni}$ was carried out using an electrolyte containing $300 \mathrm{~g} / \mathrm{L} \mathrm{NiSO}_{4}, 46 \mathrm{~g} / \mathrm{L} \mathrm{NiCl}_{2}$ and $40 \mathrm{~g} / \mathrm{L}$ $\mathrm{H}_{3} \mathrm{BO}_{3}$ at a constant $\mathrm{DC}$ voltage of $-1 \mathrm{~V}(v s . \mathrm{Ag} / \mathrm{AgCl})$ at room temperature under constant stirring. The templates were then rinsed with deionized water and Co was electrodeposited at room temperature using a bath containing $250 \mathrm{~g} / \mathrm{L} \mathrm{CoSO}_{4}$ and $40 \mathrm{~g} / \mathrm{L} \mathrm{H}_{3} \mathrm{BO}_{3}$ at a constant DC voltage of $-1 \mathrm{~V}(v s . \mathrm{Ag} / \mathrm{AgCl})$ under constant stirring. Two types of segmented nanowires of $26 \mu \mathrm{m}$ length and $80 \mathrm{~nm}$ diameter with a varying number of segments were electrodeposited. The first type of nanowire consists of one segment of $\mathrm{Co}$ and $\mathrm{Ni}$, each with a length of 13 $\mu \mathrm{m}$. From here onwards, this type of nanowire is referred to as a two-segmented nanowire (Fig. 1 (a)). The second type of nanowire consists of $750 \mathrm{~nm}$ long alternating segments of Co and $\mathrm{Ni}$ referred to as a multisegmented nanowire (Fig. 1 (b)).

\section{B. Fabrication of Electrodes onto the Nanowires}

In order to probe the magnetization reversal process of the nanowires, electrical contacts had to be patterned onto individual nanowires to perform MR measurements. For this purpose, nanowires were chemically released from the AAO template and then dispersed onto a $\mathrm{Si} / \mathrm{SiO}_{2}$ substrate. The position of an isolated and defect free nanowire was marked using etch patterns created by a Scanning Electron Microscope (SEM) equipped with a focused ion beam source. The etch patterns serve as alignment marks for the subsequent 
lithography steps [7]. To fabricate the electrodes, a bilayer photoresist approach was utilized consisting of the LOR 5B and AZ 5214 resists, which were spin coated to a thickness of $600 \mathrm{~nm}$ and $1.4 \mu \mathrm{m}$ respectively. The resists were then heated at $180^{\circ} \mathrm{C}$ and $110^{\circ} \mathrm{C}$ for 7 and 1 minutes, respectively. The spin coated sample was then exposed with a direct-writing lithography (DWL) system (Heidelberg Instruments uPG501) which utilizes a $390 \mathrm{~nm}$ LED source. This LED-based

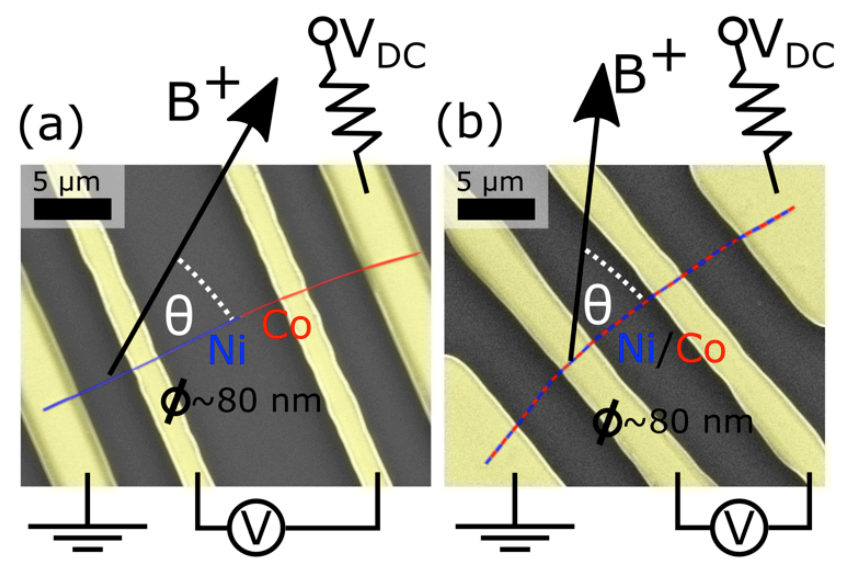

Fig. 1. SEM images with EDX overlapping of $\mathrm{Co} / \mathrm{Ni}$ nanowires connected to four electrodes: (a) two-segmented nanowire and (b) multisegmented nanowire. Electrical circuits and angular reference for the applied magnetic field are depicted schematically.

lithography is a time and cost effective process as it offers a 1000 times faster exposure compared to electron beam lithography. Even though the resolution is limited to $1 \mu \mathrm{m}$, the technique is well suited for fabricating electrodes onto single nanowires of at least $10 \mu \mathrm{m}$ length. The exposed sample was then developed using the developer AZ 726 for 1 minute after which the development process was terminated by dipping the developing sample into deionized water. The developed samples were then sputtered with $\mathrm{Cr}$ and $\mathrm{Au}$ to a thickness of $10 \mathrm{~nm}$ and $180 \mathrm{~nm}$ respectively. Prior to sputtering the samples, an etch step was performed at a pressure of $10^{-7} \mathrm{~T}$ to ensure that the $\mathrm{Cr} / \mathrm{Au}$ layer formed a low resistance electrical contact with the nanowire. Finally lift-off was performed using a resist stripper Remover PG for 10 minutes at $65^{\circ} \mathrm{C}$. The sample was then rinsed with Isopropyl Alcohol and deionized water and finally dried with $\mathrm{N}_{2}$. Fig. 1 depicts SEM images with overlapped Energy Dispersive X-ray (EDX) analysis mapping of two-segmented (Fig. 1 (a)) and multisegmented (Fig. 1 (b)) Co/Ni nanowires.

\section{Magnetoresistance Measurement Setup}

The nanowire-based device was wire bonded onto a custom built chip carrier and placed in an aluminum shield box which was connected to a DC stepper motor to enable angular rotation of the sample. The setup was then placed between the poles of an electromagnet, such that at $0^{\circ}$ orientation of the nanowire, the magnetic field was applied parallel to the nanowire axis (see Fig. 1 for reference of the angular orientation $\theta$ ).

Prior to performing the MR measurements, the nanowire was saturated by applying a magnetic field of $250 \mathrm{mT}$ along the nanowire in both directions. The MR measurements were performed at room temperature by injecting a constant current of $100 \mu \mathrm{A}$ through the outer electrodes and the voltage was measured at the inner electrodes using a nanovoltmeter while an in-plane external magnetic field was applied in steps of $0.5 \mathrm{mT}$. Once an MR measurement was performed, the orientation of the nanowire with respect to the applied field was changed in increments of $\theta \approx 1^{\circ}$ and the next MR measurement was performed at this angle. Angular MR measurements were carried out at $-90^{\circ} \leq \theta \leq 90^{\circ}$.

\section{RESULTS}

\section{A. Magnetization Reversal in Cylindrical Nanowires}

The magnetization reversal in cylindrical nanowires has been previously reported using MR measurements [9]-[12]. The MR effect is a consequence of the orientation of the magnetic moments with respect to the direction of applied electric current. The MR curve is characterized by reversible and irreversible parts and the characteristic features of an MR curve are: (1) a high resistance state, which indicates the parallel alignment of magnetic moments along the direction of current; (2) gradual decrease in resistance, which indicates the rotation of the magnetic moments away from the direction of current; (3) abrupt resistance change or a single step-like change in resistance, which is a consequence of the reversal of magnetization by DW motion and (4) finally, a high resistance state indicating complete reversal of the magnetic moments in the opposite direction.

\section{B. Magnetization Reversal in Two-Segmented Nanowires}

Segmented $\mathrm{Co} / \mathrm{Ni}$ nanowires have been reported to pin a DW at the interfaces of the segments [7], [8]. The pinning is manifested as an additional step-like resistance change in the MR curve. The pinned DW subsequently propagates only upon further increase of the applied field [7]. The MR curves consequently demonstrate a switching field $B_{s w}$, at which a part of the nanowire is reversed and a DW gets pinned, followed by a depinning field, at which the pinned DW propagates.

In this paper, magnetization reversal in both two- and multisegmented nanowires has been investigated. The twosegmented $\mathrm{Co} / \mathrm{Ni}$ nanowires offer a simplified version of the multisegmented $\mathrm{Co} / \mathrm{Ni}$ nanowires as they comprise of only one interface, which could act as a reliable pinning site. In addition, since an interface is positioned in between the inner electrodes, interfacial pinning is expected only in the electrically probed area, whereas in a multisegmented nanowire, DWs could be pinned outside the probed area (i.e. outside the inner electrodes), which would be exhibited as a higher switching field value in the MR curve.

MR measurements were performed on two-segmented $\mathrm{Co} / \mathrm{Ni}$ nanowires with the external magnetic field applied at $\theta$ $=0^{\circ}, 22.5^{\circ}, 45^{\circ}$, and $67.5^{\circ}$ with respect to the nanowire axis as depicted in Fig. 2 (a). The MR measurements reveal that a DW pins at all angles of the applied field. An additional pinning event is observed at $\theta=45^{\circ}$, which is an indication of a DW pinning that occurs at a defect within the nanowire such as a tilted interface between the Co and Ni segment. Since the pinning is observed only at a certain angle, i.e. at $45^{\circ}$, it indicates that the defect's pinning potential is dependent on 

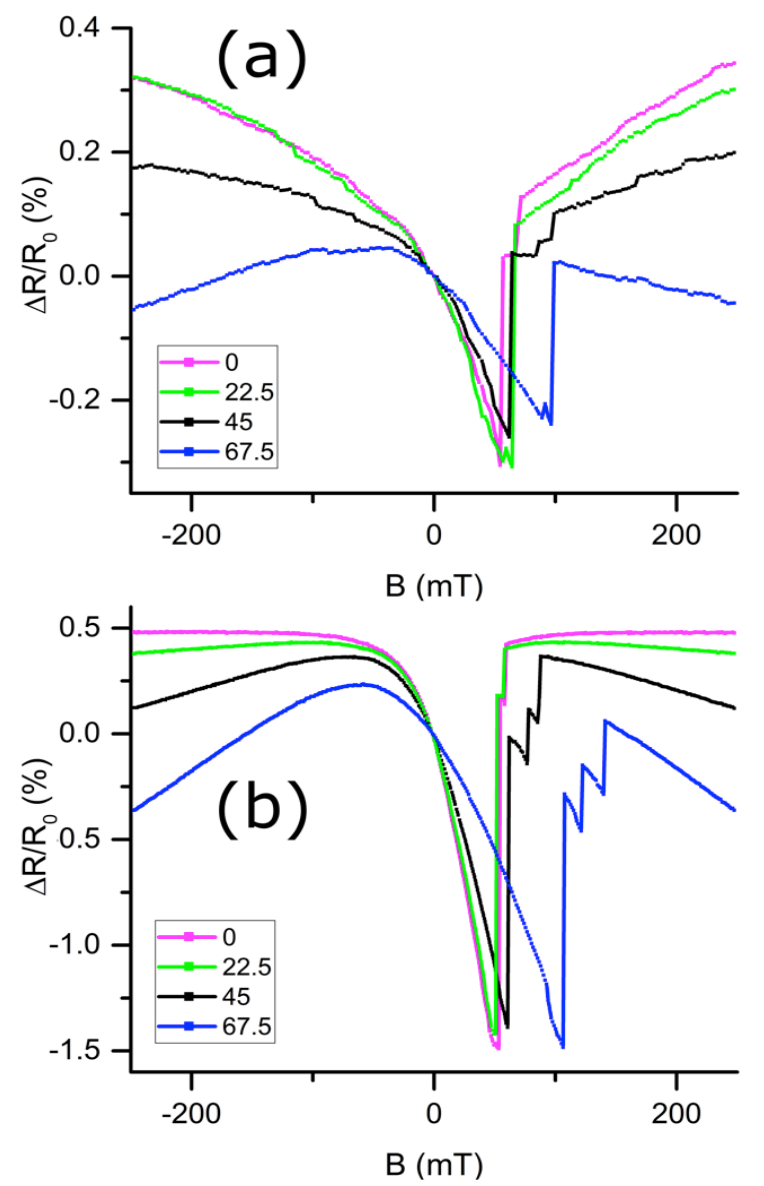

Fig. 2. MR curves of (a) two-segmented and (b) multisegmented nanowires for $\theta=0^{\circ}, 22.5^{\circ}, 45^{\circ}$, and $67.5^{\circ}$.

the magnetic field orientation. For example, the defect is energetically shallow and unable to pin the DW at $\theta=0^{\circ}$, $22.5^{\circ}$, and $67.5^{\circ}$. In addition to this, the switching field of the two segmented nanowire increases with an increased angle of the applied field. At $\theta=0^{\circ}$, the applied magnetic field acts along the nanowire axis, whereas at higher angles, the in-plane component of magnetization decreases. At $\theta=67.5^{\circ}$, the nanowire experiences a lower component of applied field along the nanowire's axis, thus requiring a higher magnitude of applied field to switch the nanowire's magnetization.

\section{Magnetization Reversal in Multisegmented Nanowires}

Fig. 2 (b) depicts the MR curves of a multisegmented $\mathrm{Co} / \mathrm{Ni}$ nanowire with external magnetic field applied at $\theta=0^{\circ}, 22.5^{\circ}$, $45^{\circ}$, and $67.5^{\circ}$ with respect to the nanowire axis. At $\theta=0^{\circ}$ and $22.5^{\circ}$, a two-step resistance change is observed in the MR curve indicating a DW pinning event. The pinned DW subsequently depins upon the application of a higher magnitude of applied field. The DW is no longer expected to pin at the subsequent interfaces, as now the in-plane component of magnetization experienced by the DW is larger than the pinning field. A different scenario is observed at higher angles, i.e. at $\theta=45^{\circ}$ and $67^{\circ}$, where a three-step resistance change is observed, indicating two pinning events. This implies that after the first pinning event, the DW propagates and gets pinned at another interface, since the in- plane field experienced by the DW is lower at higher angles of the applied field.

\section{Field Induced Domain Wall Depinning}

In order to investigate whether the steps in the MR curve are a consequence of a pinned DW, MFM was performed. Fig. 3 depicts the MFM images obtained from a multisegmented nanowire with a magnetic field applied at $\theta=22.5^{\circ}$. The blue and red dashed boxes in the MFM images indicate the $\mathrm{Ni}$ and Co segments, respectively, as identified by EDX analysis. The nanowire was firstly saturated by an applied field of $-90 \mathrm{mT}$. The saturated nanowire in stage I displays alternating dark and bright contrasts at the interface of the segments, which are a consequence of the stray fields emanating from the Co segments [8]. The applied field is gradually reversed, however, the direction of the nanowire magnetization remains

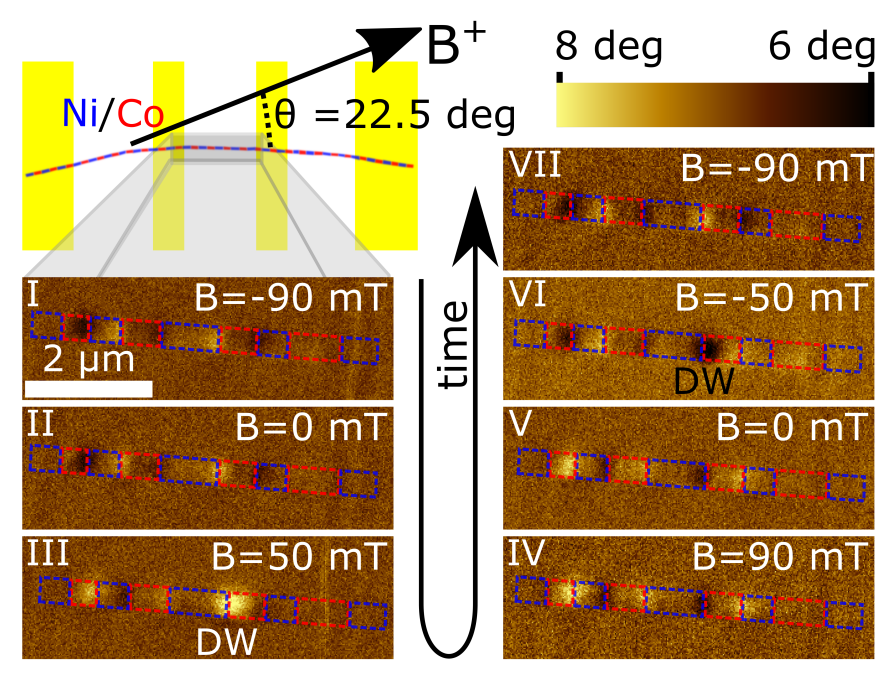

Fig. 3. Schematic diagram of multisegmented $\mathrm{Co} / \mathrm{Ni}$ nanowire from Fig. 1a; MFM images (I to VII) with applied field B (at $\theta=22.5^{\circ}$ ). The blue and red dashed boxes indicate the $\mathrm{Ni}$ and Co sections from EDX overlapping. DW pinning is observed in stages III and VI.

largely unchanged in remanence, as observed in stage II. The magnitude of the field is gradually increased and at stage III, at $\mathrm{B}=50 \mathrm{mT}$, the stray field contrasts emanating from the Co segments appear reversed. In addition to this, a DW is pinned at one of the $\mathrm{Co} / \mathrm{Ni}$ interfaces. Further increase in the field results in the depinning and propagation of the DW in the direction of the applied field leading to the saturation of the nanowire in the reverse direction at $\mathrm{B}=90 \mathrm{mT}$ as observed in stage IV. The measurement was repeated by sweeping the magnetic field from $\mathrm{B}=+90 \mathrm{mT}$ to $-90 \mathrm{mT}$. The direction of magnetization within the nanowire remains unchanged at remanence as depicted in stage V. In stage VI, a DW pinning is again observed at the same interface as in stage III followed by a depinning and reversal of the nanowire's magnetization as observed in stage VII. Thus, the second step in resistance at $\mathrm{B}=53 \mathrm{mT}$ and $\theta=22.5^{\circ}$ in Fig. 2 (b) can be attributed to the presence of a pinned DW. Furthermore, at $\theta=22.5^{\circ}$ only a single pinning event is observed in the MFM images, as is the case in the MR measurements. 


\section{E. Angular Dependence of Switching Field}

Angular dependence studies have been previously reported in $\mathrm{Co}, \mathrm{Ni}$, and $\mathrm{Co}_{\mathrm{x}} \mathrm{Ni}_{\mathrm{x}}$ nanowires [10]-[13]. The value of the switching field $B_{S W}$ as well as its dependence on the angle between the applied field and nanowire axis gives an insight into the magnetization reversal mechanism within the nanowire. Reversal mechanism in finite cylindrical nanowires has been proposed to be a localized curling mode, where an open vortex area is nucleated at the end of the nanowire and a DW propagates and consequently reverses the magnetization
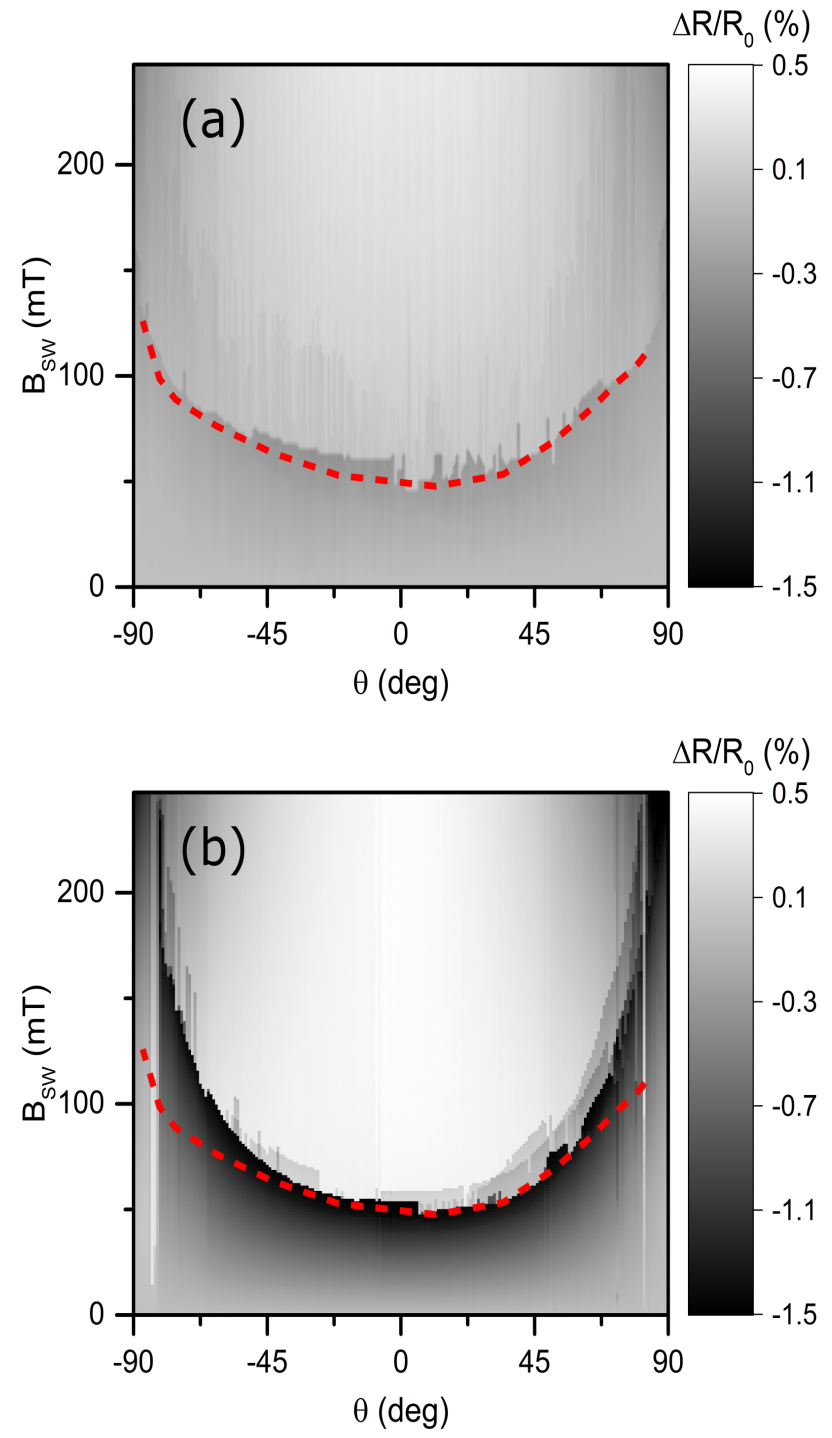

Fig. 4. Angular mapping of MR for (a) two-segmented nanowire and (b) multisegmented nanowire. Dashed lines in both graphs indicate switching field of the two-segmented nanowire.

within the nanowire. The dependence of the nanowire switching field $B_{S W}$ with respect to the field angle $\theta$ was determined for two-segmented and multisegmented $\mathrm{Co} / \mathrm{Ni}$ nanowires with a diameter of $80 \mathrm{~nm}$ using angular MR measurements as shown in Fig. 4 The dotted red line in Fig. 4 indicates the switching field of the two-segmented nanowire. The general trend in the angular dependence curve is that as the magnitude of $\theta$ increases, the switching field increases in both types of nanowires, which is characteristic for the localized curling reversal mode in a finite cylinder. The switching field of the two-segmented and multisegmented nanowires are relatively close to each other at low angles of the applied field, whereas a significant deviation in their switching field is observed at higher angles, where the reversal of the multisegmented nanowire occurs at higher applied fields. This can be due to a DW, which is pinned outside the probed area leading to a higher switching field in the multisegmented nanowire.

\section{CONCLUSION}

Segmented Co/Ni nanowires have been demonstrated to pin domain walls between their interfaces. In order to utilize this interfacial pinning phenomenon, it is crucial to gain an understanding of the magnetization reversal within these nanowires. Magnetoresistance measurements as well as the angular dependence of the nanowire switching field gives an insight into the magnetization reversal process. In this paper, two types of $\mathrm{Co} / \mathrm{Ni}$ nanowires namely multisegmented and two-segmented nanowires have been studied. The twosegmented nanowires offer a simplified structure to investigate interfacial pinning.

Pinning of a DW at the $\mathrm{Co} / \mathrm{Ni}$ interface was demonstrated by means of MR measurements in both types of nanowires. In addition to this, DW pinning was also demonstrated in multisegmented nanowires using MFM. Angular MR measurements of the multisegmented nanowire reveal an increase in the number of pinning events with an increased angle of the applied field. Even though the angular dependence of the switching field of both nanowires are similar at low angles of the applied field, a characteristic difference is observed at higher angles, where the twosegmented nanowires display a low switching field value. The above observations are a consequence of the low in-plane component of magnetization experienced by a pinned DW at higher values of $\theta$, as well as the possibility of a DW pinned outside the probed area leading to a higher observed switching field in multisegmented nanowires. These observations contribute towards the understanding of the angular switching field in segmented nanowires which is crucial for a race track memory device. We observe that a higher sensitivity to the detection of domain walls can be observed by orienting the external field at an angle to the nanowire. By aligning the field parallel to the nanowire, it is possible to trigger all the DW depinning events at once, whereas by changing the angular orientation of the field, it is possible to distinguish clearly between different DW depinning events and hence change locally the magnetization of the nanowire thereby allowing to selectively operate different areas of a racetrack memory.

\section{ACKNOWLEDGMENT}

This work was funded partly by King Abdullah University of Science and Technology and in part by EMRP and EMRP participating countries under Project EXL04 (SpinCal), and FP7 project NanoMag, and NanoMag (EMPIR). 


\section{REFERENCES}

[1] S. S. P. Parkin, M. Hayashi, and L. Thomas, "Magnetic domain-wall racetrack memory," Science, vol. 320, no. 5873, pp. 190-194, Apr. 2008.

[2] S. Song et al., "Three-Dimensional Integration of Organic Resistive Memory Devices," Advanced Materials, vol. 22, pp. 5048, 2010.

[3] F-P. Amalio et al., "Three dimensional magnetic nanowires grown by focused electron-beam induced deposition," Scientific Reports, vol. 3 , March 2013.

[4] H. Masuda and K. Fukuda, "Ordered metal nanohole arrays made by a two-step replication of honeycomb structures of anodic alumina," Science, vol. 268, no. 5216, pp. 1466, Jun. 1995.

[5] M. Yan, A. Kákay, S. Gliga, and R. Hertel, "Beating the Walker limit with massless domain walls in cylindrical nanowires," Phys. Rev. Lett., vol. 104, pp. 057201, Feb. 2010.

[6] L. Rodríguez et al., "Quantitative Nanoscale Magnetic Study of Isolated Diameter-Modulated FeCoCu Nanowires," ACS Nano, vol. 10, pp. 9669, 2016.

[7] H. Mohammed, E. V. Vidal, Y. P. Ivanov, and J. Kosel, "Magnetotransport Measurements of Domain Wall Propagation in Individual Multisegmented Cylindrical Nanowires," IEEE Trans. Magn., vol. 52, no. 7, 2016.

[8] Y. P. Ivanov, A. Chuvilin, S. Lopatin, and J. Kosel, "Modulated magnetic nanowires for controlling domain wall motion: towards $3 \mathrm{D}$ magnetic memories," ACS Nano, vol. 10, pp. 5326, 2016.

[9] A. Fert and L. Piraux, "Magnetic nanowires," J. Magn. Magn. Mater., vol. 200, pp. 338, Oct. 1999.

[10] J-E. Wegrowe, D. Kelly, A. Franck, S. E. Gilbert, and J.-Ph. Ansermet, "Magnetoresistance of Ferromagnetic Nanowires," Physical Review Letters, vol. 82, no. 18, pp. 3681, May 1999.

[11] S. Pignard et al., "Study of the magnetization reversal in individual nickel nanowires," Journal of Applied Physics, vol. 87, no. 2, pp. 824, 2000.

[12] Y. Rheem, B-Y. Yoo, W. P. Beyermann and N.V Myung, "Electro- and magneto-transport properties of a single CoNi nanowire," Nanotechnology, vol. 18, pp. 125204, 2007.

[13] W. Wernsdorfer et al., "Nucleation of Magnetization Reversal in Individual Nanosized Nickel Wires," Physical Review Letters, vol. 77, no. 9, Aug. 1996. 